\title{
IDEAL BOUNDARIES OF A RIEMANN SURFACE FOR THE EQUATION $\Delta u=P u$
}

\section{J. L. SCHIFF}

\begin{abstract}
For a nonnegative density $\boldsymbol{P}$ on a hyperbolic Riemann surfaces $R$, let $\Delta^{P}$ be the subset of the Royden harmonic boundary consisting of the nondensity points of $\boldsymbol{P}$. This ideal boundary, as well as the $\boldsymbol{P}$-harmonic boundary $\delta_{P}$ of the $P$-compactification of $R$, have been employed in the study of energy-finite solutions of $\Delta u=P u$ on $R$. We show that $\Delta^{P}$ is homeomorphic to $\delta_{P}-\left\{s_{P}\right\}$, where $s_{P}$ is the $P$-singular point. It follows that $\delta_{P}$ fails to characterize the space $\operatorname{PBE}(R)$ in the sense that it is possible for $\delta_{P}$ to be homeomorphic to $\delta_{Q}$, but $\operatorname{PBE}(R)$ is not canonically isomorphic to $Q B E(R)$.
\end{abstract}

1. In order to study the space $P B E(R)$, or $P E(R)$, for a density $P \geqslant 0$ on a hyperbolic Riemann surface $R$, two ideal boundaries have been especially suited to the task. One is the subset $\Delta^{P}$ of the Royden harmonic boundary $\Delta$, consisting of the nondensity points of $P$, and the other, $\delta_{P}$, is the $P$-harmonic boundary. of the $P$-compactification of $R$. The former was introduced in [1], and the latter in [6]. An interesting feature of $\delta_{P}$ is the existence of the $P$-singular point $s_{P}$, which in a sense corresponds to $\Delta \backslash \Delta^{P}$.

The question naturally arises as to what is the relation between $\Delta^{P}$ and $\delta_{P}$, and in this note we prove that $\Delta^{P}$ and $\delta_{P}-\left\{s_{P}\right\}$ are homeomorphic.

In [2], it was shown that $\Delta^{P}$ does not characterize $P B E(R)$ in the sense that there exist densities $P$ and $Q$ on $R$ with $\Delta^{P}=\Delta^{Q}$, but $P B E(R)$ is not canonically isomorphic to $Q B E(R)$. Here, a canonical isomorphism is a vector space isomorphism $\psi: \operatorname{PBE}(R) \rightarrow Q B E(R)$ such that for each $u \in$ $P B E(R),|u-\psi u| \leqslant p_{u}$, for some potential $p_{u}$ on $R$. Equivalently, $u \mid \Delta=$ $\psi u \mid \Delta$, for every $u \in P B E(R)$. As a consequence of the homeomorphism between $\Delta^{P}$ and $\delta_{P}-\left\{s_{P}\right\}$, we will see that $\delta_{P}$ suffers from a similar limitation in being able to characterize $P B E(R)$.

2. Let $R$ be a hyperbolic Riemann surface, and $M(R)$ the Royden algebra of bounded, Tonelli, Dirichlet-finite functions on $R$. Denote by $M_{\Delta}(R)$ the $B D$-closure of functions in $M(R)$ with compact supports. The Royden compactification $R^{*}$ is a compact Hausdorff space containing $R$ as an open dense subset such that every $f \in M(R)$ has a continuous extension to $R^{*}$.

Received by the editors December 6, 1976.

AMS (MOS) subject classifications (1970). Primary 30A50.

Key words and phrases. Hyperbolic Riemann surface, Royden harmonic boundary, nondensity points, $\boldsymbol{P}$-harmonic boundary, $\boldsymbol{P}$-singular point, canonical isomorphism, energy-finite solutions of $\Delta u=P u$. 
Moreover, $M(R)$ separates points of $R^{*}$.

The Royden boundary is $\Gamma=R^{*} \backslash R$, and the Royden harmonic boundary $\Delta$ can be characterized by

$$
\Delta=\left\{x \in R^{*}: f(x)=0 \text { for all } f \in M_{\Delta}(R)\right\},
$$

and hence is compact. Refer to the monograph [7] for a comprehensive treatment of the Royden algebra and Royden compactification.

For a nonnegative locally Hölder continuous differential $P=P(z) d x d y$, $z=x+i y$, we consider the $P$-algebra $M_{P}(R)$ of bounded, Tonelli, energyfinite functions $f$ on $R$, where the energy $E_{R}(f)$ is given by

$$
E_{R}(f)=\int_{R}|\operatorname{grad} f|^{2}+\int_{R} P f^{2}
$$

Let $M_{P \Delta}(R)$ be the family of functions which are $B E$-closures of functions in $M_{P}(R)$ with compact supports. The $P$-compactification $R_{P}^{*}$ is a compact Hausdorff space containing $R$ as an open dense subset such that every $f \in M_{P}(R)$ has a continuous extension to $R_{P}^{*}$. Furthermore, $M_{P}(R)$ separates points of $R_{P}^{*}$.

The $P$-boundary is $\gamma_{P}=R_{P}^{*} \backslash R$, and the $P$-harmonic boundary $\delta_{P}$ can be characterized by

$$
\delta_{P}=\left\{x \in R_{P}^{*}: f(x)=0 \text { for all } f \in M_{P \Delta}(R)\right\} .
$$

The $P$-singular point $s_{P} \in \delta_{P}$ enjoys the property that $f\left(s_{P}\right)=0$ for each $f \in M_{P}(R)$. It exists, and is unique, if and only if $\int_{R} P=\infty$. Moreover, under the condition $\int_{R} P=\infty, M_{P}(R) \varsubsetneqq M(R)$ and $R_{P}^{*} \neq R^{*}$.

Of prime importance in the sequel will be the following:

URYSOHN PROPERTY FOR $R^{*}$ (resp. $R_{P}^{*}$ ): For disjoint compact subsets $K_{0}, K_{1}$ of $R^{*}$ (resp. $R_{P}^{*}$ such that $K_{0}$ contains the $P$-singular point $s_{P}$ ), there exists a function $f \in M(R)$ (resp. $\left.M_{P}(R)\right)$ such that $0 \leqslant f \leqslant 1$ on $R^{*}$ (resp. $R_{P}^{*}$ ), and $f \mid K_{i}=i, i=0,1$.

Denoting by $P B E(R)$ the space of bounded, energy-finite solutions of $\Delta u=P u$ on $R$, the orthogonal decomposition obtains for every $f \in M_{P}(R)$ :

$$
f=u_{f}+g
$$

where $u_{f} \in P B E(R)$ is the $P$-harmonic projection of $f$, and $g \in M_{P \Delta}(R)$. The $P$-algebra and $P$-compactification have been extensively investigated in the works [4], [5], [6], and from a somewhat different point of view in [3].

Next, we can define a continuous open mapping $\pi_{P}: R^{*} \rightarrow R_{P}^{*} \subset$ $\Pi_{f \in M_{P}(R)}\left[-\|f\|_{\infty},\|f\|_{\infty}\right]$ of $R^{*}$ onto $R_{P}^{*}$ by $\left(\pi_{P}(x)\right)_{f}=f(x)$, for $x \in R^{*}$, $f \in M_{P}(R)$. Then $\pi_{P}$ is the identity mapping on $R$, and $f\left(\pi_{P}(x)\right)=f(x)$ for all $x \in R^{*}, f \in M_{P}(R)$.

In what follows, we prove that $\delta_{P}-\left\{s_{P}\right\}$ is the image under $\pi_{P}$ of the set of nondensity points $\Delta^{P} \subset \Delta$, defined by

$$
\Delta^{P}=\left\{x \in \Delta: \int_{U^{*} \cap R} P<\infty \text { for some neighborhood } U^{*} \text { of } x \text { in } R^{*}\right\} .
$$


3. Points of $\Gamma$ which are mapped to $s_{P}$ by $\pi_{P}$ have the following characterization (cf. [8]).

THEOREM 1. $\pi_{P}(x)=s_{P}$ if and only if $\int_{U^{*} \cap R} P=\infty$ for every neighborhood $U^{*}$ of $x$ in $R^{*}$.

Proof. Suppose $\pi_{P}(x)=s_{P}$ for some $x \in \Gamma$, and let $U^{*}$ be a neighborhood of $x$ in $R^{*}$. By the Urysohn Property for $R^{*}$, there exists a function $g \in M(R)$ such that $0 \leqslant g \leqslant 1$ on $R^{*}, g(x)=1$, and supp $g \subset U^{*}$. Then $g \in M_{P}(R)$ since every $f \in M_{P}(R)$ satisfies $f(x)=f\left(s_{P}\right)=0$. As a consequence, $E_{R}(g)=\infty$, and therefore $\int_{R} P g^{2}=\infty$ since $g \in M(R)$. Hence

$$
\int_{U^{*} \cap R} P \geqslant \int_{U^{*} \cap R} P g^{2} \geqslant \int_{R} P g^{2}=\infty,
$$

and the necessity is proved.

On the other hand, suppose $\pi_{P}(x)=y \neq s_{P}$. We choose a function $f \in$ $M_{P}(R)$ such that $f(y)>\varepsilon$ for some $\varepsilon>0$. Then $N^{*}=\left\{z \in R_{P}^{*}: f(z)>\varepsilon\right\}$ is an open neighborhood of $y$ in $R_{P}^{*}$ with

$$
\varepsilon^{2} \int_{N^{*} \cap R} P \leqslant \int_{N^{*} \cap R} P f^{2} \leqslant E_{R}(f)<\infty .
$$

Consider a neighborhood $U^{*}$ of $x$ in $R^{*}$ such that $\pi_{P}\left(U^{*}\right) \subset N^{*}$. Since $U^{*} \cap R \subset \pi_{P}\left(U^{*}\right) \cap R$, we conclude

$$
\int_{U^{*} \cap R} P \leqslant \int_{\pi_{P}\left(U^{*}\right) \cap R} P \leqslant \int_{N^{*} \cap R} P<\infty,
$$

establishing the theorem.

Corollary. If $\Delta^{P} \varsubsetneqq \Delta$, then $\pi_{P}\left(\Delta \backslash \Delta^{P}\right)=\left\{s_{P}\right\}$.

The relationship between $\Delta$ and $\delta_{P}$ was initially studied in [9]. The following result establishes the relationship between $\Delta^{P}$ and $\delta_{P}-\left\{s_{P}\right\}$.

THEOREM 2. $\pi_{P}: \Delta^{P} \rightarrow \delta_{P}-\left\{s_{P}\right\}$ is a homeomorphism.

Proof. We first show that $\pi_{P}\left(\Delta^{P}\right) \subset \delta_{P}-\left\{s_{P}\right\}$. Let $x \in \Delta$, and consider a function $f \in M_{P \Delta}(R)$. Since $M_{P \Delta}(R) \subset M_{\Delta}(R), f(x)=0$ by the characterization of $\Delta$, and since $f(x)=f\left(\pi_{P}(x)\right)$, we infer $\pi_{P}(x) \in \delta_{P}$. Therefore $\pi_{P}(\Delta) \subset \delta_{P}$. For any $x \in \Delta^{P}$, Theorem 1 implies $\pi_{P}(x) \neq s_{P}$, so that $\pi_{P}\left(\Delta^{P}\right)$ $\subset \delta_{P}-\left\{s_{P}\right\}$.

To prove $\pi_{P}$ is injective, take $x, y \in \Delta^{P}, x \neq y$. Then $\left\{\pi_{P}(x)\right\} \cup\left\{\pi_{P}(y)\right\}$ is a compact subset of $\delta_{P}-\left\{s_{P}\right\}$. By the Urysohn Property for $R_{P}^{*}$, there exists a function $g \in M_{P}(R)$ such that $0 \leqslant g \leqslant 1$ on $R_{P}^{*}$, and $g \mid\left\{\pi_{P}(x)\right\} \cup\left\{\pi_{P}(y)\right\}$ $=1$. Thus $g \mid\{x\} \cup\{y\}=1$. Choose $h \in M(R)$ such that $h(x) \neq h(y)$, and $0 \leqslant h \leqslant 1$. The function $f=g h$ satisfies $f(x) \neq f(y)$ and $0 \leqslant f \leqslant g$ on $R$. Hence $f \in M_{P}(R)$, and $f\left(\pi_{P}(x)\right) \neq f\left(\pi_{P}(y)\right)$ implies $\pi_{P}(x) \neq \pi_{P}(y)$.

To show $\pi_{P}$ is surjective, suppose $x \in \delta_{P}-\left\{s_{p}\right\} \backslash \pi_{P}(\Delta)$. By the Urysohn 
Property for $R_{P}^{*}$, there exists a function $f \in M_{P}(R)$ such that $0 \leqslant f \leqslant 1$ on $R_{P}^{*}, f(x)=1$, and $f \mid \pi_{P}(\Delta) \cup\left\{s_{p}\right\}=0$. Then the $P$-harmonic projection $u_{f} \in$ $\operatorname{PBE}(R)$ satisfies $u_{f}\left|\delta_{P}=f\right| \delta_{P}$. Thus $u_{f}(x)=1$, but $u_{f}|\Delta=u| \pi_{P}(\Delta)=0$, implying $u_{f} \equiv 0$ by the $P B D$-Maximum Principle (cf. [2]). This contradicts $u_{f}(x)=1$, and hence $\delta_{P}-\left\{s_{P}\right\} \subset \pi_{P}(\Delta)$. For any $z \in \Delta \backslash \Delta^{P}$, Theorem 1 implies $\pi_{P}(z)=s_{P}$, so that $\delta_{P}-\left\{s_{P}\right\} \subset \pi_{P}\left(\Delta^{P}\right)$, and the theorem is proved.

From the preceding it is evident that:

COROLlaRY 1. $\pi_{P}(\Delta)=\delta_{P}-\left\{s_{P}\right\}$ if and only if $\Delta=\Delta^{P}$.

Corollary 3.3 of [2] can now be stated in the following manner:

Corollary 2. $H B D(R)$ is canonically isomorphic to $\operatorname{PBE}(R)$ if and only if $\pi_{P}(\Delta)=\delta_{P}-\left\{s_{P}\right\}$.

4. A necessary condition for $\operatorname{PBE}(R)$ to be canonically isomorphic to $Q B E(R)$ is that $\Delta^{P}=\Delta^{Q}$ (cf. [2]). In this case, $\pi_{Q} \circ \pi_{P}^{-1}$ is a homeomorphism between $\delta_{P}-\left\{s_{P}\right\}$ and $\delta_{Q}-\left\{s_{Q}\right\}$. Since $\delta_{P}$ and $\delta_{Q}$ are compact, we can extend $\pi_{Q}{ }^{\circ} \pi_{P}^{-1}$ to a homeomorphism between $\delta_{P}$ and $\delta_{Q}$, yielding:

THEOREM 3. If $P B E(R)$ is canonically isomorphic to $Q B E(R)$, then $\delta_{P}$ is homeomorphic to $\delta_{Q}$.

To disprove the converse, consider the Riemann surface $T^{\infty}$ constructed in [2] which has the property that for certain densities $P$ and $Q$ on $R, \Delta^{P}=\Delta^{Q}$, however, $P B E\left(T^{\infty}\right)$ is not canonically isomorphic to $Q B E\left(T^{\infty}\right)$. The densities $P$ and $Q$ satisfy $\int_{R} P=\int_{R} Q=\infty$, so that $R_{P}^{*} \neq R^{*}, R_{Q}^{*} \neq R^{*}$. Thus we see that $\delta_{P}$ does not characterize the space $\operatorname{PBE}(R)$ in the following sense:

THeOREM 4. There exists a Riemann surface $R$ and densities $P$ and $Q$ on $R$ such that $\delta_{P}$ is homeomorphic to $\delta_{Q}$, but $\operatorname{PBE}(R)$ is not canonically isomorphic to $Q B E(R)$.

\section{REFERENCES}

1. M. Glasner and R. Katz, On the behavior of $\Delta u=P u$ at the Royden boundary, J. Analyse Math. 22 (1969), 345-354.

2. M. Glasner and M. Nakai, The roles of nondensity points, Duke Math. J. 43 (1976), 579-595.

3. Y. K. Kwon and L. Sario, The P-singular point of the P-compactification for $\Delta u=P u$, Bull. Amer. Math. Soc. 77 (1971), 128-133.

4. Y. K. Kwon, L. Sario and J. Schiff, Bounded energy-finite solutions of $\Delta u=P u$ on $a$ Riemannian manifold, Nagoya Math. J. 42 (1971), 95-108.

5 . The P-harmonic boundary and energy-finite solutions of $\Delta u=P u$, Nagoya Math. J. 42 (1971), 31-41.

6. M. Nakai and L. Sario, $A$ new operator for elliptic equations and the P-compactification for $\Delta u=P u$, Math. Ann. 189 (1970), 242-256.

7. L. Sario and M. Nakai, Classification theory of Riemann surfaces, Springer-Verlag, Berlin, Heidelberg and New York, 1970.

8. J. L. Schiff, Relations between boundaries of a Riemannian manifold, Bull. Austral. Math. Soc. 6 (1972), 25-30. 
IDEAL BOUNDARIES OF A RIEMANN SURFACE FOR THE EQUATION $\Delta u=P u \quad 61$

9. C. Wang, Quasibounded P-harmonic functions, Doctoral Dissertation, Univ. of California, Los Angeles, 1970.

Department of Mathematics and Computer Science, Western Washington State College, Bellingham, Washington 98225

Current address: Department of Mathematics, University of Auckland, Auckland, New Zealand 